\title{
Matters of life and death: long-term mortality after nonvariceal upper gastrointestinal bleeding
}

$\mathrm{P}$ atients who have had a nonvariceal upper gastrointestinal bleed (UGIB) have higher long-term mortality rates than age-matched and sex-matched controls, according to the findings of a recent study published in PLoS Medicine. Although the findings of this study are perhaps not entirely surprising, it is interesting to note that deaths from all causes, not just gastrointestinal causes, were increased after a UGIB.

Colin Crooks and co-workers, who performed the study, have previously demonstrated that patients with comorbidities unrelated to the gastrointestinal tract have an increased risk of a UGIB. "We therefore hypothesized that as sicker patients are more likely to have a UGIB, any longterm higher rates of mortality could be due to the fact they were sicker before the bleed," explains Crooks. "In some patients a UGIB would therefore be like a hip fracture, which can be a marker of an underlying deterioration in health."

Even though a UGIB is fairly common, the long-term outcomes for patients who have experienced a nonvariceal UGIB are not well understood; prior studies have investigated outcomes, but most of these focused on 30-day mortality rates, or were small in size or are now quite old. Crooks and colleagues performed a large cohort study investigating the causes of death in the 5 years after a UGIB; patients were compared with age-matched and sex-matched controls. "To have the power to look at individual causes of death, we used linked routine electronic databases of primary and secondary care in the Clinical Practice Research Datalink, which have been linked to the UK Office for National Statistics death registry; the latter provides a standardized reporting of cause of death from death certificates," says Crooks.

16,355 patients who had a nonvariceal UGIB between 1997 and 2010 were matched to 81,523 controls. The researchers used a statistical method (cumulative incidence functions) to calculate the absolute excess risk of death for each cause-because each cause of death is a competing risk for all other causes of death, cumulative incidence functions are able to adjust for this bias.

The total 5-year risk of death due to gastrointestinal causes (malignant or nonmalignant) ranged from $3.6 \%$ (patients $\leq 50$ years) to $15.2 \%$ (patients $\geq 80$ years), representing an excess risk when compared with controls of between $3.6 \%$ and $13.4 \%$, respectively; the total 5-year risk of death due to a nongastrointestinal cause ranged from $4.1 \%$ ( $\leq 50$ years) to $46.6 \%$ ( $\geq 80$ years), representing an excess risk of between $3.8 \%$ and $19.0 \%$, respectively. The largest absolute increases in risk of death were from neoplastic and cardiovascular disease. Notably, however, half of the patients who died from these two causes were not diagnosed prior to the UGIB.

Comparing these findings with outcomes after other acute medical events is also interesting. For example, threequarters of deaths after a myocardial infarction are found to be caused by the cardiovascular disease itself. Crooks and colleagues have demonstrated that this observation is not the case for a UGIB: more than half of the excess risk of death was a result of an unrelated comorbidity.

"The strength of the present study is the careful assessment of the cause of death by cross-referencing different databases," says Marc Bardou,

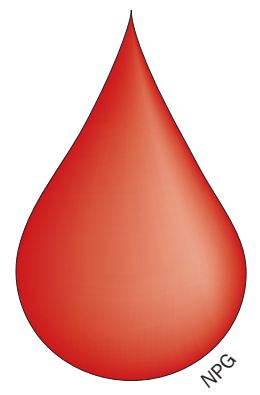

who has been involved in conducting international consensus conferences for acute nonvariceal upper gastrointestinal bleeding but was not involved in the current study. Bardou is unsure whether the paper will have a substantial effect on patient care. "However, the impact it might have on future research might be to assess the effect of a UGIB in the natural course of a disease and to conduct trials to define, for example, if initial management of patients with a UGIB has to differ according to the underlying condition," he told Nature Reviews Gastroenterology \& Hepatology.

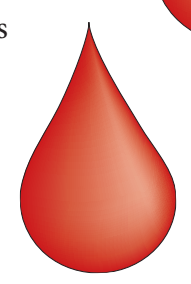

The researchers believe that the strengths of their study are its large size, length of follow-up, competing risk adjustment and general population setting. They recognize that some limitations do exist though, such as potential misclassification of risk factors for excess mortality. Nonetheless, Crooks concludes that, "In clinical practice, after a patient has had a UGIB treated, it is then important to think outside the upper gastrointestinal tract and ask the question: could the UGIB be a marker of an underlying deterioration in health either from a known comorbidity or from a new disease process?"

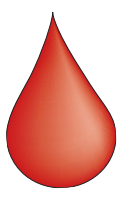

Isobel Leake

Original article Crooks, C. J. et al. Excess long-term
mortality following non-variceal upper gastrointestinal
bleeding: a population-based cohort study. PLoS Med.
10, e1001437.
Further reading Crooks, C. J. et al. Comorbidities affect
risk of nonvariceal upper gastrointestinal bleeding.
Gastroenterology doi:10.1053/j.gastro.2013.02.040 |
Bardou, M. et al. Diagnosis and management of nonvaricea
upper gastrointestinal bleeding. Nat. Rev. Gastroenterol.
Hepatol.9,97-104 (2012).

Original article Crooks, C. J. et al. Excess long-term mortality following non-variceal upper gastrointestina 10, e1001437. Bardou, M. et al. Diagnosis and management of nonvariceal Hepatol. 9, 97-104 (2012). 